\title{
Der historische Machtwechsel in Japan vor dem Hintergrund von Wahlrecht und Wählerverhalten
}

\author{
Carmen Schmidt
}

Bei den Wahlen zum japanischen Unterhaus am 30. August 2009 konnte die bis dahin stärkste Oppositionspartei, die Demokratische Partei Japans (DPJ, Nihon minshu tô), die seit 1955 nahezu ununterbrochen herrschende konservative Liberaldemokratische Partei (LDP, Jiyu minshu tô) als Regierungspartei ablösen und als stärkste Fraktion in das japanische Parlament einziehen. Damit kam es zum ersten Mal seit mehr als einem halben Jahrhundert zu einem wirklichen Machtwechsel in Japan, sieht man einmal ab vom kurzfristigen Machtverlust der LDP im Jahre 1993. ${ }^{1}$ Die jetzige Regierungspartei DPJ ist eine relativ neue politische Kraft, die erst im Jahr 1998 als Zusammenschluss von Dissidenten der LDP und der damals größten Oppositionspartei, der Sozialdemokratischen Partei Japans (SDPJ, Nihon shakai minshu tô) entstand. Die DPJ umfaßt seither verschiedene ideologische Strömungen eines Spektrums von konservativ bis progressiv. Die ideologischen Unterschiede in ihren Reihen sind daher größer als in anderen japanischen Parteien. ${ }^{2}$

Bemerkenswert ist allerdings nicht nur die Tatsache, dass die LDP nunmehr die Macht an eine neue politische Kraft abgeben musste, sondern auch die Höhe des Wahlerfolgs der DPJ und der immensen Verluste der LDP, die zuvor gemeinsam mit ihrem Koalitionspartner Kômeitô, dem politischen Arm der buddhistischen Sekte Sôka gakkai, eine Zweidrittelmehrheit besaß. Auch hatte die LDP in der Vergangenheit stark vom bestehenden Wahlsystem profitiert.

\section{1. Überblick über das japanische Parteiensystem}

Im Jahr 1955 fanden mit der Vereinigung der beiden Flügel der Sozialistischen Partei Japans (SPJ, Nihon shakai tô), der späteren SDPJ ${ }^{3}$, und dem Zusammenschluss der beiden konservativen Parteien zur Liberal-Demokratischen Partei die entscheidenden politischen Fusionen statt, die das japanische Parteiensystem in grundlegender Weise bis 1993 geprägt haben. Daneben blieb vorerst nur die Kommunistische Partei Japans (KPJ, Nihon kyôsan tô) als wenn auch noch relativ unbedeutende - Partei bestehen. Damit wurde eine Zeit permanenter Umstrukturierungen im Parteiensystem beendet und das so genannte 1955er-System mit der LDP als Regierungspartei und der SPJ als stärkster Oppositionspartei etabliert.

Die Polarisierung der beiden politischen Lager verminderte sich im Zuge des raschen ökonomischen Wachstums der 1960er Jahre. Eine Folge dieser Entwicklung war eine Zunahme der Bevölkerung in den städtischen Metropolen und ein damit verbundener sozialer

1 Im Anschluss war die LDP zwar zu wechselnden Koalitionen gezwungen, doch stellte sie ab 1996 wieder durchgehend den Ministerpräsidenten.

2 Vgl. Masaki Taniguchi, Shûgiin giin no seisaku ichi [Die Politikpositionen von Unterhausmitgliedern], in: Nihon seiji kenkyû, 3. Jg. (2006), H. 1, S. 90 - 108.

3 Im Jahr 1986 hatte die Partei ihr marxistisch geprägtes Programm durch eine sozialdemokratische Programmatik ersetzt und sich in Sozialdemokratische Partei Japans (SDPJ) umbenannt. Anschließend wurde die Partei bedeutungslos. 
und berufsstruktureller Wandel, der zu einer Abnahme der traditionellen konservativen Wählerschicht und damit zu stetigen Stimmenverlusten der LDP führte. Da sich die SPJ jedoch weiterhin fast ausschließlich auf die gleichfalls abnehmende Zahl der gewerkschaftlich organisierten Arbeiter und Angestellten stützte, schrumpften auch ihre Unterstützergruppen. Diese Prozesse begünstigten eine Pluralisierung des Parteiensystems, die sich in der Entstehung von DSP (Demokratisch-Sozialistische Partei; Minshu shakai tô) und Kômeitô als neuen Parteien der Mitte niederschlug.

Angesichts der mit der Abnahme der Kernwählerschaften einhergehenden Erosion von Wählerbindungen kann seit Anfang der 1970er Jahre - ebenso wie in anderen Industriestaaten - ein „Auftauen” des Parteiensystems beobachtet werden. Die Entwicklungen seit Beginn der 1990er Jahre zeigen, dass diese dealignment-Trends die Instabilität von Parteiensystemen begünstigen. Zu Anfang dieses Jahrzehnts kam es zur Spaltung der LDP aufgrund von Machtkämpfen innerhalb der Partei ohne deutlich erkennbare programmatische beziehungsweise interessenbezogene Differenzen. Diese Spaltung löste eine Reihe von Neugründungen aus, die zu einem fragmentierten, sich permanent umstrukturierenden Oppositionslager führten, wobei vor allem drei Prozesse von Bedeutung waren: (1) die Auflösung der Mehrzahl der bestehenden Oppositionsparteien und die Gründung der Shinshintô (Neue Fortschrittspartei) 1994; (2) die Spaltung der SDPJ 1996, die zu ihrer Bedeutungslosigkeit führte; (3) die Auflösung der Shinshintô und in der Folge die Neuformierung des Parteiensystems 1997/98 mit der Gründung der Demokratischen Partei, die anschließend zur stärksten Oppositionspartei wurde (vgl. Abbildung 1). Auch nach dieser vorläufigen Stabilisierung ist das Parteiensystem weiterhin von ständigen Auflösungen und Neugründungen geprägt. ${ }^{4}$

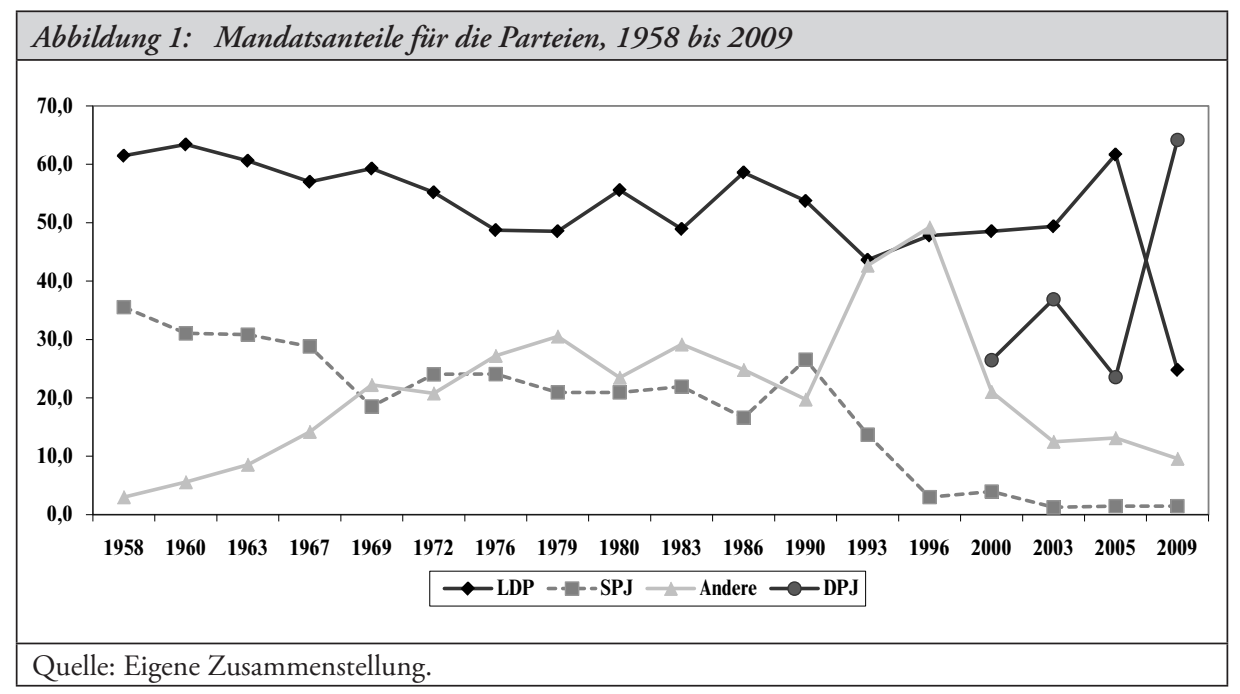

4 Zu den Entwicklungen im japanischen Parteiensystem vgl. Carmen Schmidt, Sozialstruktur und politisches System in Japan. Gesellschaftliche Konfliktlinien, politische Repräsentation und die Formierung der Wählerschaft, Marburg 2001; dies., Japan's New Party System. Characteristics and Future Perspectives, in: Roland Czada / Kenji Hirashima (Hrsg.), Germany and Japan after 1989. Reform Pressures and Political System Dynamics, Tokio 2009, S. $1-7$. 


\section{Die Unterhauswahl 2009 und der Machtwechsel}

An der Unterhauswahl 2009 beteiligten sich neben DPJ und LDP die drei etablierten Parteien, Kômeitô, SDPJ und KPJ. Des Weiteren traten auch einige neue Parteien an. Drei von ihnen waren bereits vor der Unterhauswahl 2005 gegründet worden: die Neue Volkspartei (Kokumin shintô) und die Neue Partei „Nippon“ (Shintô Nippon), bestehend aus Dissidenten der LDP, die die Partei aus Protest gegen die Postprivatisierungspläne des damaligen Ministerpräsidenten Koizumi Jun’ichirô verlassen hatte ${ }^{5}$, sowie die Neue Partei „Daichi“ (Shintô daichi), die sich ebenfalls aus ehemaligen LDP-Abgeordneten zusammensetzt. ${ }^{6}$ Die „Partei für alle" (Minna no tô) wurde 2009 kurz vor der Wahl von einem ehemaligen LDP-Minister ins Leben gerufen. Schließlich trat noch die „Partei zur Realisierung eines glücklichen Lebens" (Kôfuku jitsugen tô) zur Wahl an, eine rechts-konservative und religiöse Gruppierung.

Im Mittelpunkt des Unterhauswahlkampfes 2009 stand die Forderung der DPJ nach einem Regierungswechsel (seiken kôtai), die an das Leitmotiv Barack Obamas im Präsidentschaftswahlkampf 2008 erinnerte, der unter dem Slogan „Change“ einen radikalen Wandel versprochen hatte. Daneben versprach die DPJ in ihrem Wahlprogramm 2009 die Einführung von Kindergeld und die Schaffung gerechterer Bildungschancen, die Einführung einer Mindestrente und die Verbesserung der medizinischen Betreuung von Älteren, eine Stärkung der lokalen und regionalen politischen Autonomie sowie die Belebung des Arbeitsmarktes durch Steuervergünstigungen für kleine und mittlere Unternehmen und die Zahlung einer monatlichen Pauschale für Arbeitslose. Die Gegenfinanzierung dieser kostenintensiven Wahlversprechen sollte durch ein rigoroses Vorgehen gegen die Verschwendung von Steuergeldern für überflüssige Infrastrukturprojekte und einen Abbau der Bürokratie erfolgen. ${ }^{7}$ Die LDP hingegen stellte ihren Wahlkampf unter das Motto „Japan schützen" (Nihon o mamoru). ${ }^{8}$ Im Mittelpunkt stand die Revitalisierung der Wirtschaft; doch darüber hinaus unterschieden sich die Forderungen der LDP nur minimal von jenen der DPJ, weshalb diese der LDP „geistigen Diebstahl“ vorwarf.?

Aufgrund von Umfrageergebnissen hatten die Medien einen Wahlsieg der DPJ und eine Niederlage der LDP bereits seit dem Herbst 2008 vorhergesagt. Die Prognose trat mit erstaunlicher Präzision ein, obwohl verschiedene politische Ereignisse in den Monaten vor der Wahl potentiell die LDP hätten begünstigen sollen, so etwa der Spendenskandal, in den DPJ-Präsident Ozawa Ichirô verwickelt war und der zu seinem Rücktritt im Mai 2009 führte, sowie die von der Regierung zur Milderung der Auswirkungen der internationalen

5 Im August 2005 löste der damalige Ministerpräsident Koizumi das Unterhaus auf und schrieb Neuwahlen aus, da sein Gesetzentwurf, der die Privatisierung der japanischen Postdienste vorsah, wegen großen innerparteilichen Widerstands im Oberhaus zurückgewiesen wurde. Vor der so genannten „Postwahl“ (yûsei senkyo) verließen etliche LDP-Parlamentarier aus Protest gegen die Pläne des Ministerpräsidenten die Partei und schlossen sich neuen Gruppierungen an.

6 Gegründet von Suzuki Muneo, der die LDP bereits 2002 nach Bestechungsvorwürfen gegen seine Person verlassen hatte.

7 Siehe DPJ, The Democratic Party of Japan's Platform for Government, http://www.dpj.or.jp/ english/manifesto/manifesto2009.pdf (Abruf am 30. Oktober 2010).

8 Siehe LDP, Jimintô seisaku Bank [Wahlplattform], http://www.jimin.jp/jimin/jimin/sen_syu45/ seisaku/pdf/2009_bank.pdf (Abruf am 30. Oktober 2010)

9 The Economist, Promises, Promises: One of the World's Most Entrenched Political Parties Faces the Fight of its Life in Japan, http://www.economist.com/node/14159600?story_id=14159600 (Abruf am 18. Dezember 2009). 


\begin{tabular}{|l|c|c|c|c|c|}
\hline Tabelle 1: Ergebnis der japanischen Unterhauswahl 2009 \\
\hline \multicolumn{1}{|c|}{ Partei } & $\begin{array}{c}\text { Stimmen } \\
\text { (in Prozent) }\end{array}$ & $\begin{array}{c}\text { Differenz zu } \\
2005 \\
\text { (in Prozent) }\end{array}$ & Mandate & $\begin{array}{c}\text { Mandate } \\
\text { (in Prozent) }\end{array}$ & $\begin{array}{c}\text { Mandate, } \\
\text { Differenz zu } \\
2005 \\
\text { (in Prozent) }\end{array}$ \\
\hline DPJ & 44,9 & 11,1 & 308 & 64,2 & 40,6 \\
LDP & 32,7 & $-10,5$ & 119 & 24,8 & $-36,9$ \\
Kômeitô & 6,3 & $-1,1$ & 21 & 4,4 & $-2,1$ \\
KPJ & 5,6 & $-1,6$ & 9 & 1,9 & 0 \\
SDPJ & 3,1 & $-0,3$ & 7 & 1,5 & 0 \\
Kokumin shintô & 1,4 & 0,2 & 3 & 0,6 & $-0,2$ \\
Minna no tô & 2,6 & 2,6 & 5 & 1,0 & 1,0 \\
Shintô Nippon & 0,5 & $-0,7$ & 1 & 0,2 & 0 \\
Shintô daichi & 0,3 & 0 & 1 & 0,2 & 0 \\
Kôfuku jitsugen tô & 0,6 & 0,6 & 0 & 0 & 0 \\
Andere & 0,5 & $-1,9$ & 0 & 0 & $-3,8$ \\
Unabhängige & 1,4 & $-1,0$ & 6 & 1,3 & $-2,5$ \\
\hline N & 100 & \multicolumn{5}{|c|}{480} & 100 & \\
\hline Quelle: Eigene Berechnung nach Jiji Press vom 31. August 2009; Einerwahlkreise: http://www.jiji.com/ \\
jc/zc?k=200908/2009083101336 (Abruf am 18. Dezember 2009); Verhältniswahlkreise: http://www.jiji. \\
com/jc/zc?k=200908/2009083101337 (Abruf am 18. Dezember 2009). \\
\hline
\end{tabular}

Finanzkrise aufgelegten milliardenschweren Konjunkturprogramme. Die Probleme der LDP lagen überwiegend im personellen Bereich. Erwähnt sei hier der Rücktritt von Finanzminister Nakagawa Shôichi im Frühjahr 2009 nach einem skandalträchtigen Auftritt beim G7-Treffen in Rom und die extreme Unbeliebtheit des Ministerpräsidenten Asô Tarô, die sich allmonatlich in den Umfragen niederschlug. Selten in der Nachkriegsgeschichte Japans war ein Kabinett ähnlich unpopulär wie das Asô-Kabinett mit einer Zustimmungsrate von lediglich knapp über 20 Prozent.

Bei der Unterhauswahl erhielt die DPJ 44,9 Prozent der Stimmen, ein Plus von 11 Punkten gegenüber 2005. Aufgrund des geltenden Wahlrechts schlug sich dieser Zugewinn in einem vierzigprozentigen Zuwachs an Mandaten nieder. Die LDP hingegen erhielt nur 32,7 Prozent der Stimmen (-10,5 Punkte). Damit verlor die Partei fast 37 Prozent ihrer bisherigen Sitze. Die Kômeitô verlor leicht (-1,1 beziehungsweise -2,1 Punkte), die anderen Parteien hielten in etwa ihr Ergebnis von 2005, waren aber mit jeweils unter zwei Prozent der Mandate relativ unbedeutend. Die „Partei zur Realisierung eines glücklichen Lebens“ erhielt nur 0,6 Prozent der Stimmen und konnte keinen Sitz gewinnen (vgl. Tabelle 1).

Die Wahlbeteiligung war mit knapp 70 Prozent relativ hoch; sie lag um etwa zwei Punkte über dem Ergebnis von 2005 und um zehn Punkte über der niedrigsten Beteiligung im Jahr 1996. Diese Zahlen deuten auf eine hohe Mobilisierung von Nichtwählern hin, die offensichtlich der DPJ zugute kam. Bemerkenswert ist, dass auch prominente Politiker der ehemaligen Regierungskoalition gegen junge Kandidaten, weibliche Herausforderer und Neulinge unterlagen. ${ }^{10}$

10 So verloren etwa der ehemalige Finanzminister Nakagawa den Wahlkreis Hokkaidô 11 an einen früheren 36-jährigen Sekretär des DPJ-Generalsekretärs Ozawa Ichirô und der ehemalige Vertei- 
Die DPJ koaliert seither mit der SDPJ und Kokumin shintô, eine Zusammenarbeit, die bereits im Vorfeld der Wahl beschlossen wurde. ${ }^{11}$ Gemeinsam verfügen die Koalitionspartner und einige verbündete Unabhängige nunmehr mit 322 Mandaten über eine Zweidrittelmehrheit im Unterhaus (67 Prozent), während die vormalige Regierungskoalition aus LDP und Kômeitô nur noch 140 Abgeordnete stellt, was lediglich 26 Prozent der Sitze entspricht. Die Unterhauswahl von 2009 ergab somit eine genaue Umkehrung des Ergebnisses der vorherigen Wahl von 2005, als LDP und Kômeitô eine Zweidrittelmehrheit gewinnen und die DPJ nur etwa ein Drittel der Mandate stellen konnte.

Gemessen an der Zahl der Mandate musste die vormalige Regierungskoalition somit drastische Einbußen hinnehmen. Im Vergleich zur Wahl 2005 verlor die LDP in den Einerwahlkreisen etwa 5,2 Millionen Stimmen, in den Verhältniswahlkreisen sogar gut sieben Millionen. Allerdings hatte die Partei 2005 stark von der breiten Unterstützung für die vom damaligen Ministerpräsidenten Koizumi vorgeschlagene Postprivatisierungsreform profitiert, die in der Bevölkerung als Kampfansage gegen die „alte“ LDP-Elite aufgefasst worden war, da die Postbank in der Vergangenheit zahlreiche öffentliche Projekte finanzierte, die von etablierten Politikern der LDP zur „Wahlkreispflege“ in den eigenen Wahlkreis kanalisiert wurden.

Im Vergleich zur Unterhauswahl 2003 konnte die LDP 2009 in den Einerwahlkreisen sogar an Stimmen zulegen (+1,2 Millionen). In den Verhältniswahlkreisen verlor die Partei circa 1,8 Millionen Stimmen gegenüber 2003, was aber lediglich einem Anteil von circa zwei Prozent der Wahlberechtigten entsprach (vgl. Tabelle 2). Gemessen an den absoluten Stimmenzahlen kann das Abschneiden der LDP somit nicht als drastische Wahlniederlage betrachtet werden, da nicht zu erwarten war, dass die Partei das ungewöhnlich gute Ergebnis von 2005 würde wiederholen können. Vielmehr muss ihr Abschneiden 2009 sogar als gut bis durchschnittlich bezeichnet werden. Zu beachten ist aber, dass sich die im Vergleich zu 2003 gestiegene Stimmenzahl in den Einerwahlkreisen durch die höhere Wahlbeteiligung ganz im Unterschied zu 2005 nicht in einem wachsenden Wähleranteil manifestierte; vielmehr erreichte die Partei 2009 nur einen Stimmenanteil von 38,7 Prozent in den Einerund 26,7 Prozent in den Verhältniswahlkreisen. 2003 hatte der Anteil noch 43,8 Prozent beziehungsweise 35 Prozent betragen. Selbst 2000, als die Stimmenzahlen für die LDP deutlich unter denen des Jahres 2009 lagen, konnte sie 41 Prozent in den Einerwahlkreisen und 28,3 Prozent in den Verhältniswahlkreisen erzielen.

Die DPJ hingegen konnte sich stetig steigern und 2009 etwa doppelt so viele Stimmen wie 2000 auf sich vereinigen. Vor allem im Vergleich zur letzten Wahl im Jahr 2005 waren

digungsminister Kyûma Fumio den Wahlkreis Nagasaki 2 gegen eine erst 28-jährige DPJ-Kandidatin. Der nicht auf einem Listenplatz abgesicherte Parteivorsitzende der Kômeitô, Ôta Akihiro, verlor den Wahlkreis Tôkyô 12 gegen eine 44-jährige DPJ-Kandidatin, und auch der ebenfalls nicht auf einem Listenplatz abgesicherte Generalsekretär der Kômeitô, Fuyushiba Tetsusô, musste seinen Sitz abgeben. Damit verlor die Kômeitô ihre komplette Parteiführung. Zum ersten Mal seit 1963 musste mit Kaifu Toshiki auch ein ehemaliger Ministerpräsident seinen Wahlkreis abgeben. Vgl. Tokyo Broadcasting System, Chûmoku no senkyo ku [Beachtenswerte Wahlkreise], http://www.tbs.co.jp/senkyo2009/shu/info/chumoku.html (Abruf am 31. Oktober 2010); Mainichi Shinbun, Sôsenkyô 2009. Chûmoku no senkyo ku [Die Unterhauswahl 2009. Beachtenswerte Wahlkreise], http://mainichi.jp/select/seiji/09shuinsen/ focus/index.html (Abruf am 31. Oktober 2010).

11 Im Oberhaus koaliert die DPJ mit diesen beiden Parteien bereits seit der Wahl 2007. 
die Stimmengewinne in den Einerwahlkreisen beachtlich (+8,7 Millionen). In den Verhältniswahlkreisen lag das Stimmenplus bei circa 8,8 Millionen gegenüber 2005, was gemessen an der Zahl der Wahlberechtigten einem Zuwachs von etwa acht Punkten entsprach und sich in einem Wähleranteil von plus elf Prozent gegenüber 2005 niederschlug. Im Vergleich zur LDP hat die DPJ 2009 somit deutlich von der gestiegenen Wahlbeteiligung profitiert. ${ }^{12}$

\begin{tabular}{|l|c|c|c|c|c|c|}
\hline Tabelle 2: Absolute Stimmenzahl und prozentualer Stimmenanteil an Wablberechtigten und \\
Wählern von LDP und DPJ in den Einer- und Verhälniswahlkreisen, 2000 bis 2009 \\
\hline
\end{tabular}

Die Analyse der tatsächlich abgegebenen gültigen Stimmen stützt diesen Befund (vgl. Tabelle 3). Zwischen 2003 und 2009 stieg die Zahl der Wähler um circa 20 Millionen (bei etwa 100 Millionen Wahlberechtigten also um ein Fünftel). So war die Zahl der gültigen Stimmen 2005 im Vergleich zur Wahl 2003 um insgesamt 16,9 Millionen angestiegen, wobei die LDP mit einem Plus von rund 11,7 Millionen Stimmen am meisten profitieren konnte. 2009 stieg die Beteiligung weiter an, insgesamt um etwa fünf Millionen im Vergleich zu 2005. Dabei musste die LDP mit zwölf Millionen Stimmen etwa den Anteil an gewonnenen Stimmen der Wahl 2005 wieder abgeben, während die DPJ 17,5 Millionen hinzugewinnen konnte.

12 Die Diskrepanz zwischen den Stimmenanteilen in den Einer- und Verhältniswahlkreisen, der vor allem für die LDP deutlich wird, liegt im taktischen Wahlverhalten der Wähler begründet. Vgl. hierzu Abschnitt 3. 


\begin{tabular}{|c|c|c|c|}
\hline \multicolumn{4}{|c|}{$\begin{array}{l}\text { Tabelle 3: Summierte gültige Stimmen in den Einer-und Verhältniswablkreisen 2003, } 2005 \text { und } \\
2009 \text { im Vergleich }\end{array}$} \\
\hline & $\begin{array}{c}\text { Differenz } \\
2005 \text { zu } 2003\end{array}$ & $\begin{array}{c}\text { Differenz } \\
2009 \text { zu } 2005\end{array}$ & $\begin{array}{c}\text { Differenz } \\
2009 \text { zu } 2003\end{array}$ \\
\hline Gültige Stimmen gesamt & 16.870 .609 & 5.074 .573 & 21.945 .182 \\
\hline Gültige Stimmen LDP & 11.656 .676 & -12.293 .989 & -637.313 \\
\hline Gültige Stimmen DPJ & 1.931 .422 & 17.478 .921 & 19.410 .343 \\
\hline \multicolumn{4}{|c|}{$\begin{array}{l}\text { Anmerkung: Summierte gültige Stimmen in den Einer- und Verhältniswahlkreisen (Erst- und Zweit- } \\
\text { stimme). } \\
\text { Quelle: Eigene Berechnung nach Sômushô, http://www.soumu.go.jp/senkyo/senkyo_s/data/index.html } \\
\text { (Abruf am 31. Oktober 2010). }\end{array}$} \\
\hline
\end{tabular}

\section{Die Bedeutung des Wahlsystems für den Machtwechsel}

Das seit 1996 gültige Wahlrecht sieht bei einer Gesamtzahl von 480 Unterhausmandaten ein gemischtes Wahlsystem aus 300 Einerwahlkreisen und 180 Listenmandaten vor. In den Einerwahlkreisen gilt jeweils der Kandidat mit der höchsten Stimmenzahl als gewählt. Für die Listenmandate wurde das Land in elf Listenblöcke eingeteilt, wobei die Mandatsverteilung für die einzelnen Parteien über das d'Hondtsche Höchstzahlverfahren festgelegt wird. Somit verfügt der Wähler über jeweils eine Stimme für einen Kandidaten im Einerwahlkreis und eine Stimme für die Liste einer Partei, womit taktisches Wahlverhalten ermöglicht wird. Prominente Kandidaten in den Einerwahlkreisen können auf Listenplätzen abgesichert werden. Als Voraussetzung für die Anerkennung als Partei gelten eine Zweiprozenthürde und/oder die Mindestzahl von fünf gewählten Abgeordneten. ${ }^{13}$ Ähnlich wie beim vorher geltenden Wahlsystem kann kein bestimmtes politisches Repräsentationsziel erkannt werden, da die Art der Stimmenverrechnung angesichts der ungewöhnlichen Kombination von Einer- und Verhältniswahlkreisen weder Majorz noch Proporz zugerechnet werden kann. ${ }^{14}$

Da in den 300 Einerwahlkreisen - gegenüber nur 180 Listenmandaten - bereits eine Stimme mehr oder weniger über den Gewinn des Mandats entscheidet, kann es zu erdrutschartigen Verschiebungen kommen. Das Wahlrecht begünstigte in der Vergangenheit jedoch ausschließlich die LDP. So war der bias-Wert, der die Differenz von erreichter Stimmenzahl zur Mandatszahl wiedergibt, für die LDP seit der Einführung des Wahlsystems stetig angestiegen und lag bei der Unterhauswahl 2005 bei +19 Prozent, dem höchsten Wert in der Nachkriegsgeschichte für die LDP überhaupt. Erst bei der Wahl 2009 ergab sich erstmals ein Negativwert für die LDP (vgl. Abbildung 2).

Die Begünstigung der LDP hatte verschiedene Ursachen. Zu nennen wäre hier zunächst die immer wieder vertagte Anpassung der Wahlkreise an Migrationsprozesse, da in den LDP-Wählerhochburgen auf dem Land deutlich weniger Stimmen für ein Mandat benötigt wurden als in den Städten, den Hochburgen der Opposition. Zwischen 1996 und 2005 waren im bevölkerungsstärksten Wahlkreis etwa doppelt so viele Wähler verzeichnet wie im

13 Vgl. Kôshoku senkyo hô [Wahlgesetz], Kapitel 9, Artikel 86, http://www.houko.com/00/01/ S25/100A.HTM\#s9 (Abruf am 1. Oktober 2010).

14 Vgl. Carmen Schmidt, Sozialstruktur und politisches System in Japan, a.a.O. (Fn. 4), S. 126 129. 


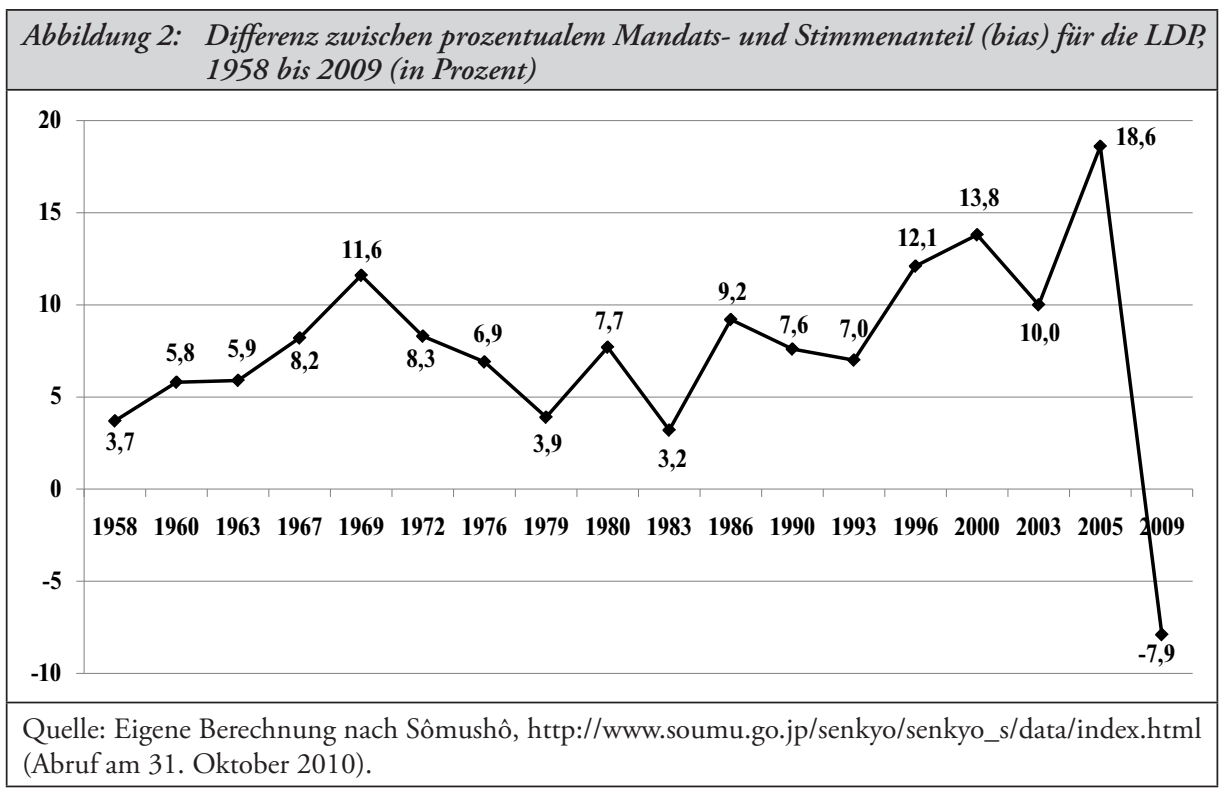

bevölkerungsschwächsten. Das Prinzip der Stimmengleichwertigkeit wurde daher grob verletzt. ${ }^{15}$ Bei der Unterhauswahl 2009 erreichte der Bevölkerungsunterschied vom kleinsten Wahlkreis Kôchi 3 zum größten Wahlkreis Chiba 4 den 2,3-fachen Wert und lag um 0,13 Prozentpunkte über der Wahl 2005. ${ }^{16}$ Die Stimmenungleichwertigkeit ist somit angestiegen und kann daher nicht den Wahlerfolg der DPJ erklären.

Darüber hinaus profitierte die LDP in der Vergangenheit von der ungewöhnlichen Kombination von Majorz- und Proporzsystem, da hierdurch ein echtes Zweiparteiensystem verhindert wurde und sich die Stimmen für die Opposition in vielen Einerwahlkreisen auf verschiedene Parteien verteilten. Da sich die Kandidaten der LDP zudem auf gut organisierte persönliche Unterstützergruppen (kôenkai) verlassen konnten, profitierte die Partei am deutlichsten von den bestehenden Regelungen. ${ }^{17}$

Dies traf 2009 nicht zu. Insgesamt konnte die DPJ elf Prozent mehr Stimmen gewinnen als 2005, was zu einem Zuwachs von 41 Prozent bei den Mandaten führte. In den Einerwahlkreisen schlug sich das Stimmenplus von elf Prozent in einem um 56 Prozent erhöhten Sitzanteil nieder. In den Verhältniswahlkreisen hingegen ergab sich erwartungsgemäß ein relativ ausgeglichenes Verhältnis zwischen Stimmen- und Mandatszuwachs von elf zu 14 Prozent. Der überwältigende Erfolg der DPJ wurde somit in den Einerwahlkreisen erzielt, in denen die LDP in der Vergangenheit deutlich mehr Sitze gewinnen konnte als andere Parteien.

15 Vgl. ebenda, S. 122 - 125.

16 Vgl. Yomiuri Shinbun, Shôsenkyoku „ippyô no kakusa” saidai de 2,305 bai [Stimmenungleichwertigkeit liegt beim bis zu 2,305-fachen Wert], http://www.yomiuri.co.jp/election/shugiin2009/ news2/20090818-OYT1T00944.htm (Abruf am 18. August 2009).

$17 \mathrm{Zu}$ den kôenkai vgl. Masumi Jun'nosuke, Contemporary Politics in Japan, Berkeley / Los Angeles / London 1995, S. $236 \mathrm{ff}$. 
Die Ursache für das gute Ergebnis der DPJ in den Einerwahlkreisen ist sowohl in der Praxis der Kandidatenaufstellung der anderen Parteien zu suchen als auch in der gestiegenen Wahlbeteiligung. Von ausschlaggebender Bedeutung dürfte vor allem gewesen sein, dass die Kommunistische Partei Japans in den Einerwahlkreisen lediglich 152 Kandidaten nominierte (vgl. Tabelle 4), nachdem es lange Zeit Praxis der KP gewesen war, überall eigene Kandidaten aufzustellen. Offiziell wurde die geänderte Strategie mit finanziellen Aspekten begründet, da Kandidaten für das Unterhaus gemäß geltendem Wahlrecht eine Kaution von sechs Millionen Yen (circa 40.000 Euro) hinterlegen müssen, die vom Staat eingezogen wird, wenn sie nicht mindestens 20 Prozent der gültigen Stimmen im Wahlkreis auf sich vereinen. Nach der Unterhauswahl 2005, in der die KPJ keinen Kandidaten in den Einerwahlkreisen durchsetzen konnte und Kautionen von 223 Kandidaten in Höhe von circa neun Millionen Euro abschreiben musste, wurden in der Partei Stimmen laut, angesichts der massiven finanziellen Einbußen einen Strategiewechsel vorzunehmen und künftig weniger Kandidaten aufzustellen. Der Verzicht zur Kandidatur dürfte in den betroffenen Wahlkreisen die DPJKandidaten begünstigt haben: Vorwahluntersuchungen zeigten, dass die KP-Wähler in diesen Fällen dem oppositionellen DPJ-Kandidaten ihre Stimme geben würden. ${ }^{18}$

\begin{tabular}{|l|c|c|c|}
\hline \multicolumn{4}{|l|}{ Tabelle 4: Kandidaten der Parteien } \\
\hline \multicolumn{1}{|c|}{ Partei } & Kandidaten 2009 & in Prozent & $+/$ - zu 2005 \\
\hline LDP & $326(289)$ & 23,7 & -20 \\
DPJ & $330(271)$ & 24,0 & 31 \\
Kômeitô & $51(8)$ & 3,7 & -1 \\
KPJ & $171(152)$ & 12,4 & -121 \\
SDPJ & $37(31)$ & 2,7 & -8 \\
Kokumin shintô & $18(9)$ & 1,3 & 4 \\
Minna no tô & $15(14)$ & 1,1 & 15 \\
Shintô daichi & $4(0)$ & 0,3 & 4 \\
Shintô Nippon & $8(2)$ & 0,6 & 0 \\
Mini Parteien & $343 *(292)$ & 25,0 & 339 \\
Unabhängige & $70(70)$ & 5,1 & 0 \\
\hline N & 1374 (1139) & 100 & 243 \\
\hline Anmerkung: In Klammern $=$ Kandidaten in den Einerwahlkreisen. ${ }^{*}=$ Enthalten sind hier die 337 Kan- \\
didaten der Kôfuku jitsugen tô. \\
Quelle: Kandidaten 2009: Asahi shinbun 2009, http://www2.asahi.com/senkyo2009/kouho/ (Abruf am \\
31. Oktober 2010); Kandidaten 2005: Asahi shinbun 2005, http://www2.asahi.com/senkyo2005/rna- \\
vi/050910_01.html (Abruf am 31. Oktober 2010); Prozentsatzdifferenz: Eigene Berechnung. \\
\hline
\end{tabular}

Zudem stimmten SDPJ und Kokumin shintô die Kandidatenaufstellung mit dem gewünschten Koalitionspartner DPJ ab. Während die DPJ zugunsten der kleineren Parteien und einigen Unabhängigen in 29 Wahlkreisen auf die Aufstellung eigener Kandidaten verzichtete, sahen die kleineren Parteien von der Aufstellung von Kandidaten in den meisten anderen Wahlkreisen ab. Auch die LDP hatte zugunsten ihres Koalitionspartner Kômeitô in acht Einerwahlkreisen auf die Aufstellung von Kandidaten verzichtet, so dass sich bei dieser Wahl erstmals in vielen Wahlkreisen $(\mathrm{N}=115)$ lediglich LDP- und DPJ-Kandidaten bezie-

18 Vgl. Sentaku Magazine, Japan Aches for a Political Rescue, in: Japan Times vom 16. Dezember 2008, S. 13. 
hungsweise von diesen beiden Parteien gestützte Bewerber gegenüber standen, womit eine Zersplitterung der oppositionellen Stimmen auf verschiedene Parteien vermieden wurde. ${ }^{19}$

Das taktische Wahlverhalten verdeutlicht Abbildung 3. Sowohl LDP als auch DPJ erzielten mehr Stimmen in den Einerwahlkreisen, während die kleineren Parteien in den Verhältniswahlkreisen deutlich mehr Stimmen auf sich vereinigen konnten. Angesichts der klaren Koalitionsaussagen vor der Wahl 2009 kann davon ausgegangen werden, dass die Wähler der Kômeitô in den Einerwahlkreisen überwiegend für den LDP-Kandidaten stimmten, die Wähler von SDPJ und Kokumin shintô hingegen für den DPJ-Bewerber, sofern kein Kandidat der eigenen Partei zur Wahl stand.

Da die KP 2009 deutlich weniger Kandidaten nominierte als bei vergangenen Wahlen, lag ihr Stimmenanteil in den Einerwahlkreisen etwa um zwei Millionen unter dem in den Verhältniswahlkreisen; 2005 war dieses Verhältnis noch ausgeglichen gewesen. Diese Stimmen standen somit anderen Bewerbern zur Verfügung. Hätten alle Wähler der KP im Einerwahlkreis für den jeweiligen DPJ-Kandidaten gestimmt, hätte dies etwa einem Viertel des $\mathrm{Zu}$ wachses der DPJ in Höhe von circa 8,7 Millionen Stimmen gegenüber 2005 entsprochen.

Zudem hatte die DPJ bei dieser Wahl deutlich von der gestiegenen Beteiligung profitiert. Mit der Zunahme der Zahl der Wähler verringert sich jedoch der Einfluss der persönlichen Unterstützergruppen der LDP-Parlamentarier, da diese vorwiegend die Gruppen der konservativen Stammwähler organisieren. Liegt die Wahlbeteiligung niedrig und treten viele Parteien in den Einerwahlkreisen an, kann die Mobilisierung dieser organisierten Gruppe bereits

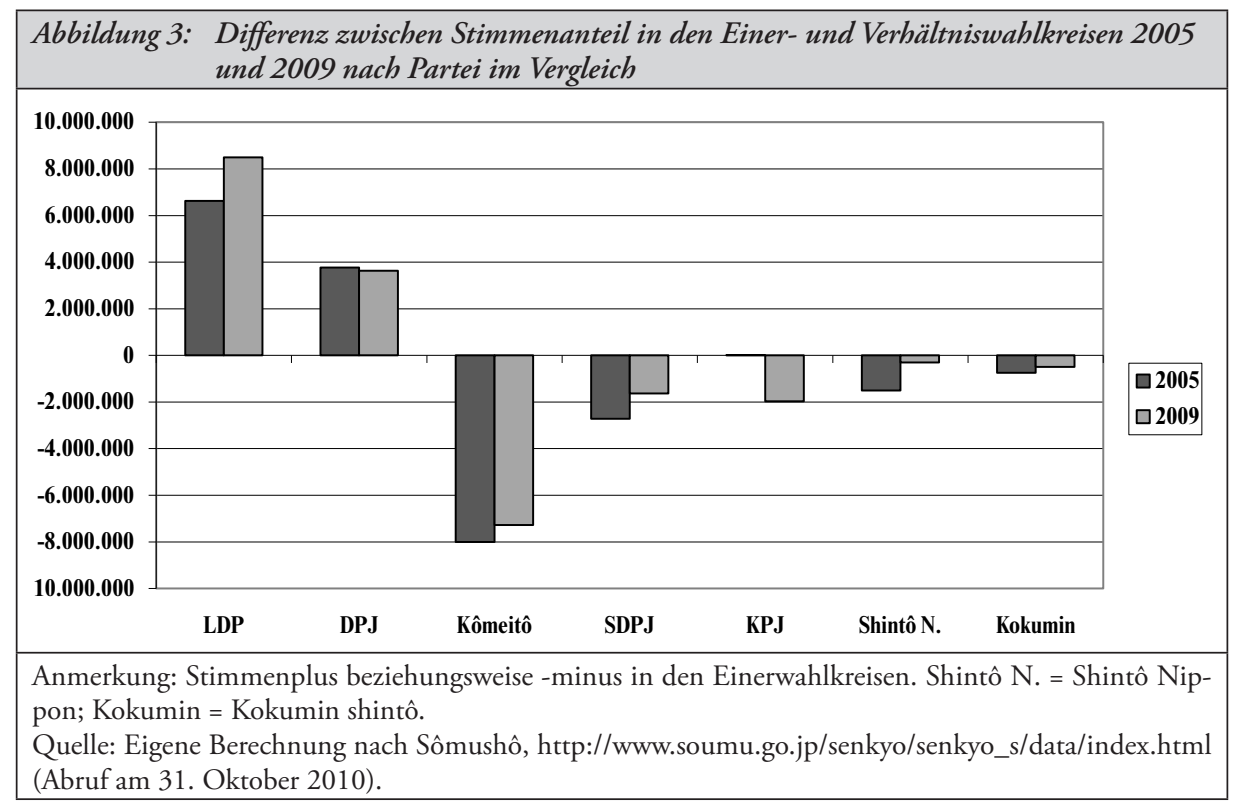

19 Eigene Auszählung nach Asahi shinbun, Sôsenkyô 2009 [Die Unterhauswahl 2009], http:// www2.asahi.com/senkyo2009/kouho/ (Abruf am 31. Oktober 2010). Ohne Berücksichtigung der Kandidaten der neugegründeten Kôfuku jitsugen tô, die zwar in fast allen Wahlkreisen eigene Kandidaten aufstellte, jedoch unbedeutend blieb. 
ausreichen, um ein Mandat zu erringen. Umgekehrt vermindert sich ihre Bedeutung, wenn die Zahl der oppositionellen Wähler steigt und nur zwei Kandidaten zur Auswahl stehen.

\section{Wablbeteiligung, Wählerverhalten und der Machtwechsel}

Die Quote der Wahlbeteiligung resultiert in Japan wie in allen Gesellschaften aus dem Zusammenwirken verschiedener Faktoren. In der Interpretation des Wahlverhaltens muss daher unterschieden werden zwischen Langzeittrends, die durch wesentliche strukturelle Determinanten hervorgerufen werden, und Kurzzeit-Fluktuationen, die durch politischkonjunkturelle Begleiterscheinungen einer bestimmten Wahl ausgelöst werden. Für den Zeitraum von 1946 bis 2009 können mehrere Phasen unterschieden werden: zwischen 1946 und 1958, als die Wahlbeteiligung stetig anstieg; von 1960 bis Anfang der 1990er Jahre mit einer stetigen Abnahme der Wahlbeteiligung, und die Phase von Mitte der 1990er Jahre bis 2003, in der die Wahlbeteiligung rapide sank. Erst bei den letzten beiden Wahlen ist wieder ein deutlicher Anstieg zu verzeichnen (vgl. Abbildung 4).

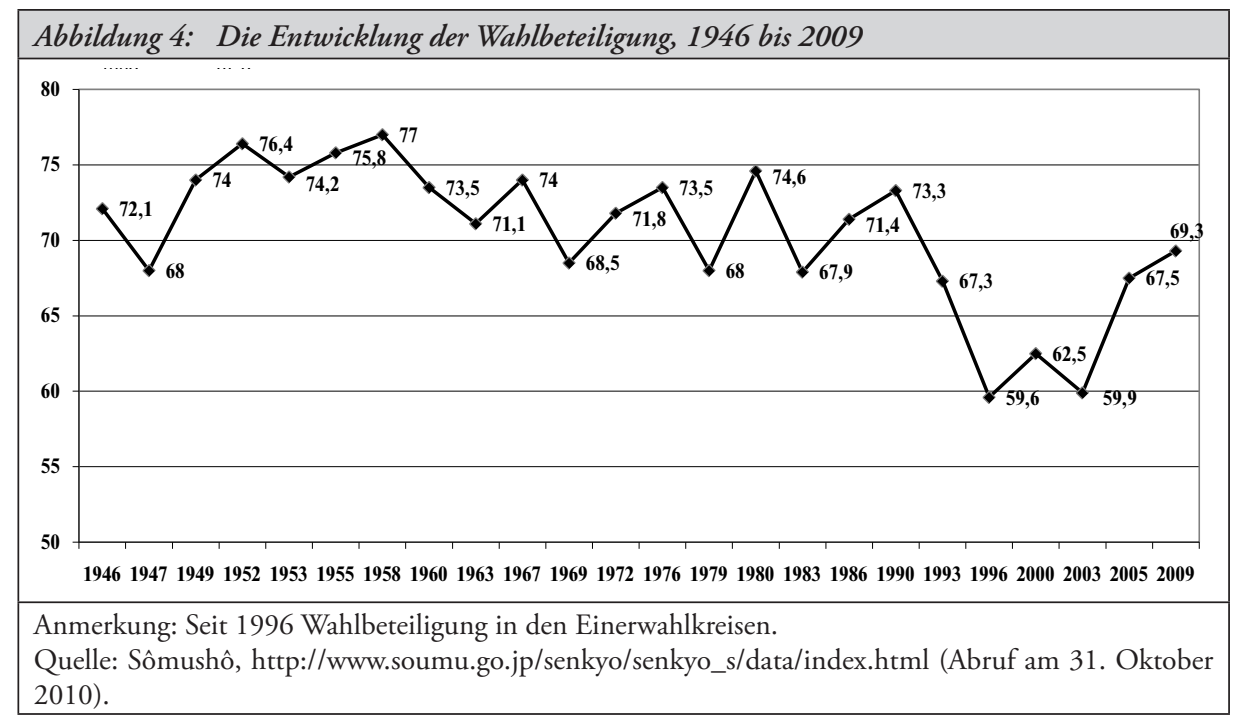

Neben der Zahl der Nichtwähler wuchs auch die Gruppe derjenigen Personen, die bei Befragungen angaben, keine Partei zu unterstützen, seit Anfang der 1970er Jahre erheblich an und blieb bis Mitte der 1980er Jahre relativ konstant. Über die 1990er Jahre zeigt die Quote erneut einen starken Anstieg und lag im Jahr 2000 bei über 50 Prozent. ${ }^{20}$

Für die Abnahme der Parteibindungen und die sinkende Wahlbeteiligung sind insbesondere zwei Prozesse verantwortlich, die in den 1960er Jahren einsetzten. Bedingt durch den sozialen Wandel nahm zum einen die Stammwählerschicht der LDP, die Gruppe der Bauern und kleinen Selbständigen, immer weiter ab; gleichzeitig bewirkte der starke Rückgang

20 Vgl. Carmen Schmidt, Sozialstruktur und politisches System in Japan, a.a.O. (Fn. 4), S. 194. 
der gewerkschaftlich Organisierten eine Reduktion der Gruppe der Stammwähler der linken Parteien. Dealignment-Trends in Form einer Ablehnung der Unterstützung von Parteien können seit den späten 1960er und 1970er Jahren allerdings vorwiegend unter denjenigen Gruppen und Schichten festgestellt werden, die in den 1950er und 1960er Jahren progressiv gewählt hatten. So setzte sich die Nichtunterstützerschicht in den 1960er Jahren noch aus den Älteren, weniger gebildeten Beschäftigten in Landwirtschaft, Forsten oder Fischerei zusammen, in den 1980er und 1990er Jahren hingegen überwiegend aus den jüngeren, besser gebildeten, abhängig Beschäftigten in den Großstädten, aus jenen Wahlberechtigten somit, aus denen die Linksparteien zuvor ihre Wählerschaft rekrutierten. Dies führte zu einer entscheidenden Schwächung der politischen Linken und schließlich zur Bedeutungslosigkeit der SDPJ. ${ }^{21}$ Der mit der Abnahme der Kernwählerschichten einhergehende dealignment-Trend begünstigte in den 1990er Jahren Instabilität und Umstrukturierungen des Parteiensystems.

Aber nicht nur der Trend zur Nichtwahl und zur Nichtunterstützung ist offenkundig, sondern auch der Wandel der ideologischen Positionen innerhalb der Bevölkerung. In Japan werden hierbei als Äquivalente zu „links“ und „rechts“ die ideologischen Positionen „progressiv“ und „konservativ“ abgefragt. Hierbei werden die Befragten aufgefordert, sich auf einer Skala von 1 bis 10 einzuordnen, wobei 1 „sehr progressiv“ und 10 „äußerst konservativ“ repräsentiert. 2009 gab fast die Hälfte an, „weder progressiv noch konservativ“ zu sein, 36 Prozent hatten eine „konservative“ oder „eher konservative“ Einstellung, und nur knapp 17 Prozent der Befragten klassifizierten sich als „progressiv“ oder „eher progressiv“. Ein Trend zur Mitte kann seit den 1970er Jahren festgestellt werden, wobei vor allem die Abnahme der Zahl derjenigen auffällt, die sich als „progressiv“ oder „eher progressiv“ einstuften und deren Anteil 1972 noch bei gut 26 Prozent gelegen hatte; und auch der Anteil der Befragten, die sich als „konservativ“ einstuften, ist seit den frühen neunziger Jahren rückläufig (vgl. Tabelle 5).

\begin{tabular}{|c|c|c|c|}
\hline \multicolumn{3}{|c|}{ Tabelle 5: } & Konservative versus progressive politische Einstellung in Japan, 1972 bis 2009 \\
\hline Jahr & $\begin{array}{c}\text { Konservativ / } \\
\text { überwiegend konservativ }\end{array}$ & Neutral / Andere / K.A. & $\begin{array}{c}\text { Progressiv / } \\
\text { überwiegend progressiv }\end{array}$ \\
\hline 1972 & 38,0 & 35,7 & 26,2 \\
1976 & 36,6 & 39,1 & 24,4 \\
1983 & 47,6 & 34,2 & 18,2 \\
1986 & 46,5 & 37,1 & 16,5 \\
1990 & 42,3 & 38,4 & 19,3 \\
1993 & 40,8 & 41,6 & 17,6 \\
1996 & 35,9 & 49,0 & 15,1 \\
2000 & 37,3 & 44,6 & 18,1 \\
2003 & 36,7 & 46,4 & 16,9 \\
2005 & 35,0 & 49,2 & 15,9 \\
2009 & 36,1 & 46,9 & 16,9 \\
\hline
\end{tabular}

Anmerkung: Antwort auf die Frage „Halten Sie sich für konservativ oder progressiv?”, K.A. = Keine Antwort.

Quelle: Akarui senkyo suishin kyōkai, jeweilige Jahrgänge, http://www.akaruisenkyo.or.jp/066search/ index.html (Abruf am 31. Oktober 2010).

21 Vgl. ebenda, S. $164-168$. 


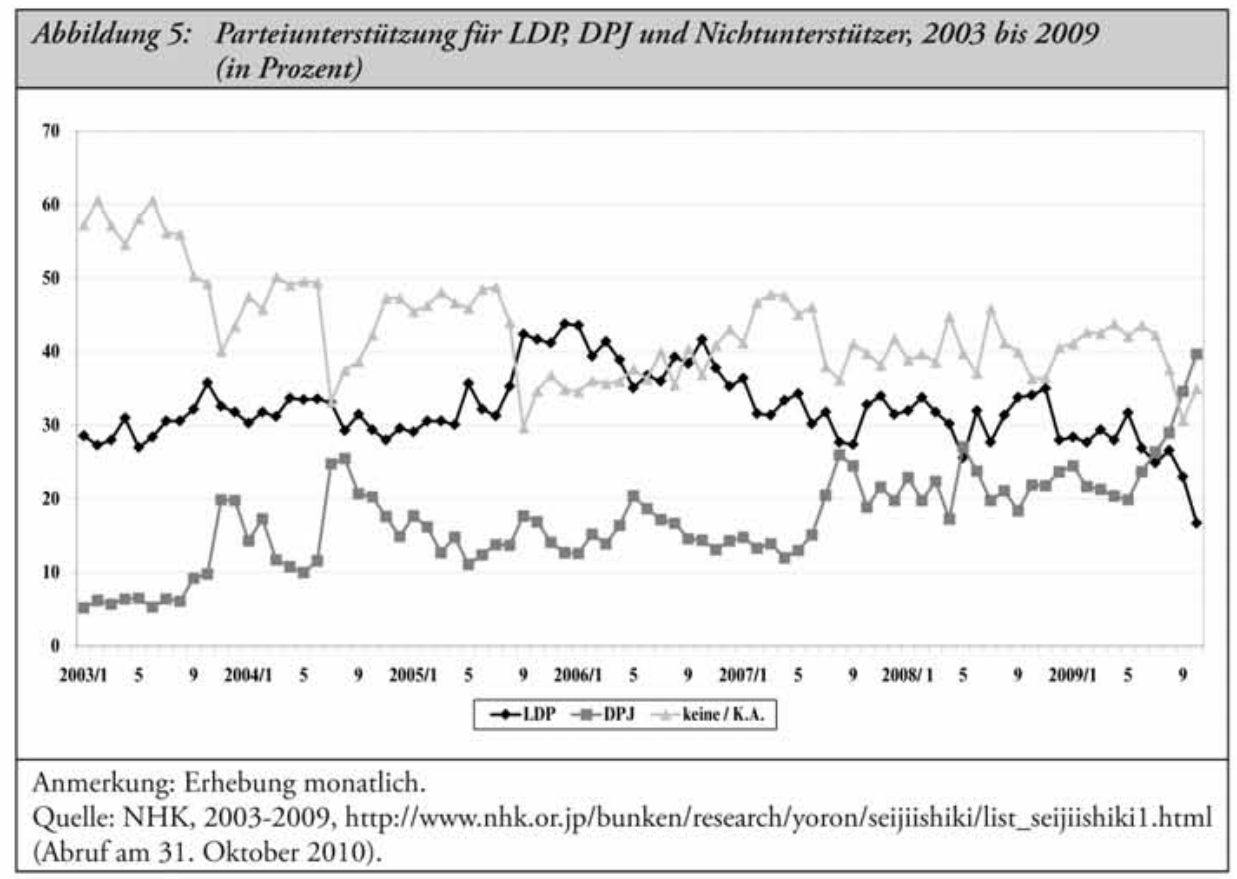

Die Abnahme der Parteibindungen und die geringe Bereitschaft, sich ideologischen Strömungen zuzuordnen, schafft ein Wählerpotential für neue Parteien wie die DPJ. Zudem steigt damit die Zahl der Wechselwähler, die frei von politischen Bindungen ihren Wahlentscheid treffen. Gemäß den Nachwahluntersuchungen von Kyôdo News gab ein Viertel der Wähler nach dem Verlassen des Wahllokals 2009 an, keine bestimmte Partei zu unterstützen. Knapp 60 Prozent dieser Befragten hatte in den Einerwahlkreisen für den DPJBewerber gestimmt, nur 23 Prozent für den LDP-Kandidaten, im Gegensatz zu gut 37 Prozent bei der Wahl von 2005. ${ }^{22}$

Angesichts nachlassender Parteibindungen kommt politischen Themen für die Mobilisierung der Wählerschaft eine immer größere Bedeutung zu. War es bei der Wahl 2005 die gesellschaftliche Polarisierung angesichts des Plans zur Privatisierung der Post des damaligen LDP-Ministerpräsidenten Koizumi, die seiner Partei zugute kam, so führte 2009 die von den Medien prognostizierte und somit vielen Wählern greifbar erscheinende Möglichkeit des Regierungswechsels zur Mobilisierung für die DPJ.

Abbildung 5 zeigt den Verlauf der Unterstützung für die Parteien von 2003 bis kurz nach der Unterhauswahl 2009. Anfang 2003 war die Zahl der Nichtunterstützer auf über 60 Prozent angestiegen. 2005 sank ihre Zahl zugunsten der LDP. Hatten noch im Juli 2005 lediglich 31 Prozent der Befragten angegeben, die Partei zu unterstützen, stieg die Quote bis auf gut 42 Prozent vor der Wahl im September, um danach wieder zu fallen. Eine ähnliche Entwicklung zeigte sich vor der Unterhauswahl 2009, nur diesmal zugunsten der DPJ. Für sie lag die Quote nach 20 Prozent im Frühjahr 2009 erst kurz vor der Wahl im

22 Vgl. Kyôdô News nach Japan Times, In Reversal from 2005. Most Swing Voters Swung to DPJ, http://search.japantimes.co.jp/cgi-bin/nn20090901a5.html (Abruf am 30. Oktober 2009). 
Sommer mit etwa 35 Prozent über der LDP-Unterstützung. Von entscheidender Bedeutung für die Zukunft der DPJ wird es daher sein, ob es ihr gelingt, diese Wähler dauerhaft an sich zu binden.

\section{Japan auf dem Weg zu einem alternierenden Zweiparteiensystem?}

Die vielfach als historisch bezeichnete Unterhauswahl in Japan hat nicht nur die mehr als ein halbes Jahrhundert fast ununterbrochen währende Herrschaft der konservativen LDP beendet, sondern auch eine relativ neue politische Partei an die Macht gebracht, die erst vor wenigen Jahren von Parlamentariern des progressiven wie konservativen Lagers ins Leben gerufen wurde.

Wie gezeigt, war das Wahlsystem für den Ausgang der Wahl eine entscheidende Determinante. So lässt sich die Diskrepanz zwischen erreichter Stimmenzahl und Mandatszahl darauf zurückführen, dass es sehr viel mehr Einerwahlkreise mit Majorzverfahren (300) als Listenmandate (180) gibt, was erdrutschartige Verschiebungen in der Sitzzahl begünstigt. Auch die Strategie der Parteien, die Kandidatenaufstellung in den Einerwahlkreisen abzustimmen, spielte eine bedeutende Rolle, da eine Zersplitterung der Oppositionsstimmen hierdurch vermieden wurde. Vor allem die Tatsache, dass die KP in zahlreichen Wahlkreisen erstmals auf die Aufstellung eigener Kandidaten verzichtete, führte dazu, dass sich in vielen Wahlkreisen nur zwei Bewerber von Regierungs- und Oppositionslager gegenüber standen.

Dealignment-Prozesse innerhalb der japanischen Wählerschaft, die weitgehend denen in anderen Industriestaaten vergleichbar sind, trugen gleichfalls zum Machtwechsel bei. Auch in Japan ist eine Lockerung der Bindungen an die großen Parteien zu beobachten, für die gesellschaftliche Wandlungsprozesse verantwortlich sind. So lässt sich eine deutliche Schrumpfung der traditionellen Wählergruppen mit der Folge eines sinkenden Einflusses dieser Bevölkerungsgruppen auf die Wahlausgänge feststellen, während die Zahl der Personen mit historisch nicht gewachsenen Parteibindungen weiter zunimmt. Diese Wählerschichten bilden ein Potential für neue Parteien wie die DPJ, deren Bemühen es sein muss, ein dauerhaftes alignment herbeizuführen.

Ausschlaggebend für den Ausgang der Wahlen 2005 und 2009 war die Mobilisierung der unentschlossenen Wähler. Vor diesem Hintergrund sind für die künftige Entwicklung des japanischen Parteiensystems mehrere Szenarien denkbar. So könnte langfristig ein System mit zwei regierungsfähigen größeren Parteien entstehen, die sich in der Regierungsverantwortung abwechseln und in dem den thematisch mobilisierten Wählern eine wichtige Rolle zukommt. Die letzten beiden Wahlen haben gezeigt, dass auch eine etablierte Partei wie die LDP mit entsprechenden Kandidaten und Themen von diesen Wechselwählern profitieren kann. Allerdings ist eine Entwicklung hin zu einem echten Zweiparteiensystem unter dem geltenden Wahlrecht in Japan schwer vorstellbar, da dieses eine Pluralisierung des Parteiensystems und die Repräsentation kleiner Parteien ermöglicht, wie die diversen Parteiabspaltungen und -neugründungen der jüngsten Vergangenheit belegen. Stabile Koalitionen zwischen zwei ideologischen Lagern sind nicht erkennbar. Vielmehr sind angesichts der großen ideologischen Differenzen innerhalb der DPJ und ihrer bislang mangelnden Verankerung in der Wählerschaft auch Abspaltungen von der Partei durchaus vorstellbar, wodurch neue Parteienkonstellationen und -koalitionen entstehen könnten und auch eine große Koalition für die Zukunft nicht ausgeschlossen werden kann. 